\title{
EDITORIAL
}

\section{TROPICAL SPRUE}

Pathology amongst tropical populations is often multiple and individual disease syndromes difficult to distinguish. Such syndromes tend to be more characteristic amongst expatriates including soldiers and this has undoubtedly been a factor in the important military contribution to tropical medical research.

The first clear description of tropical sprue was given in 1818 by Ballingal, then a Regimental Medical Officer serving in Bengal. Since then it has become apparent, that at least in soldiers, the condition is a definite entity. The onset is commonly as an "acute gastroenteritis" following eating meat. This is later succeeded by steatorrhoic diarrhoea, anorexia, loss of weight and considerable asthenia. Glossitis is a relatively late and inconstant feature. On investigation there is intestinal malaborsption of fat, xylose and vitamin B 12. Jejunal biopsy at first only reveals mild abnormality but after 3 months a characteristic morphological lesion spreads from the jejunum distally to affect the whole small gut mucosa. Convolutions and partial villous atrophy are seen under the dissecting and light microscopes respectively. Secondary progressive folate and vitamin B 12 deficiencies lead to tissue depletions of folate in 4 months and of vitamin B 12 in 3 years. During the first year there is a characteristic response to treatment with folic acid. The pathogenesis of this syndrome is so constant that a diagnosis can be made apart from a process of exclusion.

The aetiology of tropical sprue is not clear but in this military population the problem can be limited to finding the cause for the acquired susceptibility of the intestinal mucosa to folate deficiency. It is known that the local populations of endemic areas show abnormalities of jejunal structure and function as measured by Western standards. This " abnormality" does not affect expatriates living in segregated communities but did affect American Peace Corps Volunteers living in Pakistan villages. It seems likely that this "tropical enteropathy" may be associated with an increased rate of intestinal mucosal cell turnover rendering it susceptible to folate (or vitamin B 12) deficiency. When the soldier becomes exposed to this he will develop sprue, if and when he becomes folate deficient.

Tropical sprue is a disease of considerable military importance. It was the most common reason for medical invaliding of soldiers from India and Burma during World War II. The implications of recent work are clear. Folate repletion especially when supplemented by antibiotic treatment will result in a rapid clinical remission. Secondly the condition can almost certainly be prevented by ensuring a daily dietary supplement of $0.1-0.2 \mathrm{mg}$ of folic acid. Such a supplement will neither affect the efficiency of malarial antifols nor induce untoward effects in latent vitamin B 12 deficiency. 